\section{Adaptação transcultural da Escala de Independência em Atividades da Vida Diária (Escala de Katz)}

\author{
Cross-cultural adaptation of the Independence in \\ Activities of Daily Living Index (Katz Index)
}

\footnotetext{
${ }^{1}$ Escola Nacional de Saúde Pública Sergio Arouca, Fundação Oswaldo Cruz, Rio de Janeiro, Brasil. 2 Sociedade Brasileira de Geriatria e Gerontologia, Rio de Janeiro, Brasil.

Correspondência V. T. S. Lino Centro de Saúde Escola Germano Sinval Faria, Escola Nacional de Saúde Pública Sergio Arouca, Fundação Oswaldo Cruz. Rua Aristides Lobo 57, casa 10, Rio de Janeiro, $R J$ 20250-450, Brasil. valerialino@ensp.fiocruz.br
}

\begin{abstract}
This study involved the development and performance assessment of a cultural adaptation of the Katz scale of independence in activities of daily living, translated into Portuguese in Brazil. Two translations and two back-translations of the items were analyzed by experts in order to decide on the final version. Operational equivalence was assessed in a pilot study. The reliability and internal consistency of the adapted version were assessed by retesting 156 patients on the same day or 7 days after the first interview. Cultural equivalence was achieved after resolving subtle differences in some items. The final version was considered easy to understand and use. Chance-corrected agreement (weighted kappa) was 0.91. Cronbach's alpha ranged from 0.80 to 0.92. Conclusions: a Portuguese version of the Katz scale of independence in activities of daily living, thoroughly developed and tested, proved equivalent to the original version in English. The items were internally consistent and the rates were reliable.
\end{abstract}

Activities of Daily Living; Reproducibility of Results; Aged
Valéria Teresa Saraiva Lino 1,2 Silvia Regina Mendes Pereira 2 Luiz Antônio Bastos Camacho 1 Sergio Telles Ribeiro Filho 2 Salo Buksman 2

\section{Introdução}

O conceito de capacidade funcional implica a habilidade para a realização de atividades que permitam ao indivíduo cuidar de si próprio e viver independentemente 1 , constituindo-se no foco do exame do idoso e num indicador de saúde mais completo do que a morbidade, relacionando-se diretamente com a qualidade de vida 2,3 .

A capacidade funcional é medida por meio de instrumentos padronizados que avaliam o desempenho do idoso nas atividades da vida diária e nas atividades instrumentais da vida diária. Uma das escalas mais utilizadas para avaliar o desempenho nas atividades da vida diária é a Escala de Independência em Atividades da Vida Diária (EIAVD), ou Escala de Katz 4,5,6,7,8,9,10,11. Ela foi desenvolvida para a avaliação dos resultados de tratamentos em idosos e predizer o prognóstico nos doentes crônicos. A EIAVD consta de seis itens que medem o desempenho do indivíduo nas atividades de autocuidado, os quais obedecem a uma hierarquia de complexidade, da seguinte forma: alimentação, controle de esfíncteres, transferência, higiene pessoal, capacidade para se vestir e tomar banho ${ }^{4}$. Essa seqüência é semelhante à observada durante o desenvolvimento infantil, em que primeiramente a criança aprende a levar a colher à boca para, somente mais tarde, tornar-se capaz de tomar banho de forma independente. Tais considerações levaram à suposição de que a escala 
se baseia em funções primárias biológicas e psicossociais 12 .

Através de revisão da literatura, verificase que o conceito de capacidade funcional é o mesmo em diferentes culturas 13,14,15,16. No Brasil, Veras 17 demonstrou, no Rio de Janeiro, que a habilidade para realizar as atividades da vida diária era menor entre as classes de baixa renda e, tal qual em Belo Horizonte, Estado de Minas Gerais 18, diminuía com a idade. Em São Paulo, utilizando inquérito domiciliar, Rassi 19 também demonstrou que a dependência para a realização das atividades da vida diária aumentava com a idade.

No Brasil, a tradução livre da Escala de Katz vem sendo amplamente utilizada, sem ter sido realizada a adaptação transcultural, passo imprescindível antes de se conduzir um estudo e aplicar seus resultados. Esta etapa implica a análise das diferenças existentes entre as definições, crenças e comportamentos relacionados ao objeto de investigação 20 .

O tipo de tradução mais utilizado atualmente é realizado por duplas de tradutores independentes, em que se aplica o método de retradução ou back translation 21,22,23,24. A estratégia de Herdman et al. 25 recomenda, além da retradução, a análise de seis tipos de equivalência em ambas as culturas, a saber: conceitual, que ocorre quando eles apresentam a mesma idéia; itens, que se refere à adequação dos elementos da escala original para representar o conceito em questão no idioma em que o instrumento será aplicado; semântica, cujo objetivo é a transferência do significado entre as duas línguas; operacional, relativa à possibilidade de utilização de um formato semelhante de questionário, instruções, forma de administração e métodos de avaliação; de medida, cujo objetivo é verificar se as diferentes versões alcançam níveis similares em termos de validade e confiabilidade. Quando todos os tipos de equivalência forem alcançados, podese falar em equivalência funcional. Nesse caso, o instrumento medirá igualmente o conceito nas duas culturas, os resultados encontrados serão comparáveis e estará estabelecida a adaptação transcultural da nova versão.

Dada a importância do conhecimento do desempenho nas atividades da vida diária no exame de idosos e a necessidade de utilização de instrumentos adaptados para a nossa cultura, este trabalho tem por objetivo realizar a adaptação transcultural da EIAVD para a língua portuguesa, utilizando a estratégia de Herdman et al. 25, mantendo a pontuação criada pelos autores da escala em 197626 .

\section{Métodos}

Avaliação das equivalências conceitual e de itens

A revisão da literatura demonstrou a universalidade da expressão capacidade funcional, dando-se por estabelecida a equivalência conceitual, indicando que o constructo tem a mesma concepção em ambos os idiomas. Da mesma forma, observou-se que os elementos existentes na escala são pertinentes em qualquer país ou cultura, instituindo-se a equivalência de itens, pois os elementos da escala original representam o conceito em questão nas duas línguas.

\section{- Equivalência semântica}

Consistiu em quatro etapas:

1) Tradução independente do instrumento para o português por um grupo de profissionais com experiência na área da geriatria e fluentes no idioma inglês (T1) e por um profissional de nível superior bilingüe (T2);

2) T1 e T2 foram, respectivamente, retraduzidas para o inglês por um médico americano radicado no Brasil (R1) e por uma tradutora juramentada (R2), de forma independente e sem acesso ao original;

3) Uma subseqüente avaliação formal foi realizada por um epidemiologista, proficiente nos dois idiomas, que apreciou a equivalência entre o original e cada uma das retraduções (R1 e R2) sob a perspectiva do significado referencial dos termos (correspondência semântica). Utilizou-se um formulário específico, desenhado de forma a mascarar a origem dos itens da versão original e da retradução que, quando comparados, foram classificados em quatro níveis: inalterado, pouco alterado, muito alterado ou completamente alterado;

4) A equipe de pesquisadores analisou o significado geral de cada item da escala, comparando-se o que foi captado na tradução (T1 e T2) para o português com o original em inglês. Esta correspondência transcende à literalidade das palavras, encampando aspectos mais sutis, como o impacto que um termo tem no contexto cultural da população-alvo. A apreciação é necessária porque a correspondência literal de um termo não implica interpretações semelhantes em diferentes culturas. A partir de então, se propôs uma versão-síntese.

\section{- Equivalência operacional}

O pré-teste da versão proposta foi realizado por oito profissionais que trabalhavam com idosos, 
mas não utilizavam o instrumento em suas práticas. Eles examinaram 24 pacientes para avaliar a clareza do instrumento e a adequação da aplicação na sua forma de entrevista.

\section{- Equivalência de medida}

Esta etapa foi realizada em duas instituições de longa permanência da cidade do Rio de Janeiro por uma dupla de pesquisadores, sendo entrevistados auxiliares de enfermagem que assistiam os idosos em regime de plantão. Uma amostra não probabilística foi selecionada de modo a representar indivíduos para os quais a escala foi desenvolvida, sendo selecionados 100 indivíduos com 60 ou mais anos de idade. Não foram considerados elegíveis pacientes com doenças agudas graves ou em fase terminal de câncer e doenças degenerativas.

Uma outra análise foi realizada utilizando-se uma amostra de 57 indivíduos distribuídos da seguinte forma: acompanhantes de pacientes provenientes de um hospital geral foram entrevistados por uma dupla, enquanto que a outra dupla entrevistou aqueles de um ambulatório de geriatria e de uma instituição geriátrica de longa permanência. As visitas foram realizadas no mesmo dia havendo um intervalo variando entre $15 \mathrm{e}$ 60 minutos entre as avaliações.

O protocolo do estudo e o consentimento informado foram realizados de acordo com a $D e$ claração de Helsinki, de 1975, revisada em 1983, e foi aprovado pela Comissão de Ética da Sociedade Brasileira de Geriatria e Gerontologia, seção Rio de Janeiro.

\section{Análise dos dados e tamanho da amostra}

As variáveis sócio-demográficas foram consideradas na caracterização dos sujeitos da pesquisa.

A consistência interna dos itens do instrumento foi medida pelo coeficiente alfa de Cronbach estimado com dados de cada observador.

A pontuação gerada pela aplicação da escala por dois observadores foi comparada em tabela cruzada. A concordância simples foi calculada pela proporção de indivíduos em que os dois observadores obtiveram a mesma pontuação. A concordância corrigida para o acaso foi estimada pela estatística kappa 27 e kappa ponderado 28 . A concordância segundo o kappa foi classificada como ausente $(<0,0)$, fraca $(0,10-0,20)$, regular $(0,21-0,40)$, moderada $(0,41-0,60)$, substancial $(0,61-0,80)$ ou quase perfeita $(0,81-1,00) 29$. A distribuição dos dados discordantes foi analisada de modo a verificar tendenciosidade dos observadores.
Os dados foram digitados em planilha eletrônica e processados com o programa PEPI (Computer Programs for Epidemiologists; http://www. sagebrushpress.com/pepi).

O tamanho da amostra necessária para estimar a reprodutibilidade da escala foi calculado usando-se o programa nQuery Advisor versão 4.0 (Statistical Solutions, Saugus, Estados Unidos), tomando como base em cenários plausíveis de concordância corrigida (kappa ponderado). Considerando a hipótese conservadora de coeficiente de reprodutibilidade igual a $0,70 \pm 0,10$ seriam necessários aproximadamente 95 indivíduos.

\section{Resultados}

\section{Avaliação da equivalência semântica}

A apreciação do significado referencial entre os termos oriundos das retraduções e os do instrumento original revelou pouca ou nenhuma alteração em 97,8\% e 78,2\% em R2 e R1, respectivamente (Tabela 1).

Tabela 1

Avaliação da equivalência entre o original e cada uma das retraduções sob a perspectiva do significado referencial dos termos.

\begin{tabular}{lcc}
\hline Julgamento do avaliador & $\begin{array}{c}\text { T1/R1 } \\
\text { Número de itens (\%) }\end{array}$ & $\begin{array}{c}\text { T2/R2 } \\
\text { Número de itens (\%) }\end{array}$ \\
\hline Inalterado & $18(39,1)$ & $38(82,6)$ \\
Pouco alterado & $18(39,1)$ & $7(15,2)$ \\
Muito alterado & $10(21,8)$ & $1(2,2)$ \\
Completamente alterado & $0(0,0)$ & $0(0,0)$ \\
\hline
\end{tabular}

Na elaboração da versão síntese, apesar de dez itens terem sido considerados muito alterados em T1/R1 na análise do significado referencial, que avalia o sentido das palavras, cinco foram selecionados em virtude do melhor significado geral. Um exemplo disso foi a seleção do termo vaso sanitário em vez de privada, palavra considerada vulgar em relação à primeira (Tabela 2).

\section{Avaliação da equivalência operacional}

O pré-teste da versão revelou ser o instrumento de fácil aplicação e bem compreendido, tanto pelos profissionais quanto pelos idosos, não 
Tabela 2

Itens muito alterados selecionados para a versão final.

\begin{tabular}{|c|c|c|}
\hline Original & Muito alterado & Pouco alterado/Inalterado \\
\hline $\begin{array}{l}\text { Independent in feeding, continence, } \\
\text { transferring, going to toilet, dressing } \\
\text { and bathing }\end{array}$ & $\begin{array}{l}\text { Independente para alimentar-se, manter a } \\
\text { continência, transferir-se, ir ao vaso sanitário, } \\
\text { vestir-se e tomar banho }\end{array}$ & $\begin{array}{l}\text { Independente para se alimentar, se transferir, } \\
\text { usar a privada, se vestir, tomar banho e em } \\
\text { relação à continência }\end{array}$ \\
\hline Dressing & Vestir-se & Vestir-se \\
\hline $\begin{array}{l}\text { Independent: gets clothes from closets and } \\
\text { drawers; puts on clothes, outer garments, } \\
\text { braces; manages fasteners; act of tying shoes } \\
\text { is excluded }\end{array}$ & $\begin{array}{l}\text { Independente: pega as roupas dos } \\
\text { armários e gavetas, veste-as, coloca órteses } \\
\text { ou próteses, manuseia fechos. Exclui-se } \\
\text { o ato de amarrar sapatos }\end{array}$ & $\begin{array}{l}\text { Independente: apanha as roupas de armário } \\
\text { e gavetas, veste as roupas, peças externas, } \\
\text { suspensório; usa os fechos; o ato de amarrar } \\
\text { sapatos é excluído }\end{array}$ \\
\hline $\begin{array}{l}\text { Dependent: does not dress self or remains } \\
\text { partly undressed }\end{array}$ & $\begin{array}{l}\text { Dependente: veste-se apenas parcialmente } \\
\text { ou não se veste sozinho }\end{array}$ & $\begin{array}{l}\text { Dependente: não se veste sozinho ou } \\
\text { permanece parcialmente vestido }\end{array}$ \\
\hline Going to toilet & Uso do vaso sanitário & Uso da privada \\
\hline $\begin{array}{l}\text { Independent: gets to toilet; gets on and of } \\
\text { toilet; arranges clothes; cleans organs of } \\
\text { excretion (may manage on bedpan used at } \\
\text { night only and may or may not be using } \\
\text { mechanical supports) } \\
\text { Dependent: uses bedpan or commode or } \\
\text { receives assistance in getting to and using } \\
\text { toilet }\end{array}$ & $\begin{array}{l}\text { Independente: vai ao vaso sanitário, senta-se } \\
\text { e levanta-se do vaso sanitário; ajeita as } \\
\text { roupas; faz a higiene íntima (pode usar } \\
\text { comadre ou similar somente à noite e pode } \\
\text { ou não estar usando suporte mecânico) } \\
\text { Dependente: usa comadre ou similar } \\
\text { controlado por terceiros ou recebe ajuda } \\
\text { para ir até o vaso sanitário e para usá-lo }\end{array}$ & $\begin{array}{l}\text { Independente: chega até a privada; senta } \\
\text { e se levanta; arruma as roupas; limpa os } \\
\text { órgãos de excreção (pode usar o próprio } \\
\text { penico só durante a noite e pode } \\
\text { ou não estar usando suportes mecânicos) } \\
\text { Dependente: usa penico ou comadre ou } \\
\text { recebe ajuda para ir até e usar a privada }\end{array}$ \\
\hline $\begin{array}{l}\text { Continence } \\
\text { Independent: urination and defecation } \\
\text { entirely self- controlled } \\
\text { Dependent: partial or total incontinence in } \\
\text { urination or defecation; partial or total control } \\
\text { by enemas, catheters, or regulated use of } \\
\text { urinals and/or bedpan }\end{array}$ & $\begin{array}{l}\text { Continência } \\
\text { Independente: micção e evacuação } \\
\text { inteiramente autocontroladas } \\
\text { Dependente: incontinência parcial ou } \\
\text { total para micção ou evacuação; controle } \\
\text { parcial ou total por enemas, catéteres; } \\
\text { uso de urinóis ou comadre controlados } \\
\text { por terceiros }\end{array}$ & $\begin{array}{l}\text { Continência } \\
\text { Independente: urinação e defecação } \\
\text { inteiramente sob autocontrole } \\
\text { Dependente: incontinência total ou parcial } \\
\text { na urinação ou defecação; controle total } \\
\text { ou parcial por meio de enemas, catéteres, } \\
\text { ou uso regulado de urinóis e/ou penicos }\end{array}$ \\
\hline $\begin{array}{l}\text { Toileting: going to the "toilet room" for bowel } \\
\text { and urine elimination; cleaning self after } \\
\text { elimination and arranging clothes } \\
\text { () goes to the "toilet room", cleans self, } \\
\text { and arranges clothes without assistance } \\
\text { (may used object for support such as a cane, } \\
\text { walker or wheelchair and may manage night } \\
\text { bedpan or commode, emptying same in } \\
\text { morning) } \\
\text { () receives assistance in going to "toilet room" } \\
\text { or in cleansing self or in arrangin clothes after } \\
\text { elimination or in use of night bedpan or } \\
\text { commode } \\
\text { () doesn't go to room termed "toilet"for the } \\
\text { elimination process }\end{array}$ & $\begin{array}{l}\text { Uso do vaso sanitário: ida ao banheiro ou } \\
\text { local equivalente para evacuar e urinar; } \\
\text { higiene íntima e arrumação das roupas } \\
\text { () vai ao banheiro ou local equivalente, } \\
\text { limpa-se e ajeita as roupas sem ajuda } \\
\text { (pode usar objetos para apoio como bengala, } \\
\text { andador ou cadeira de rodas e pode usar } \\
\text { comadre ou urinol à noite esvaziando-o } \\
\text { de manhã) } \\
\text { () recebe ajuda para ir ao banheiro ou local } \\
\text { equivalente, ou para limpar-se, ou para } \\
\text { ajeitar as roupas após evacuação e micção, } \\
\text { ou para usar a comadre ou urinol à noite } \\
\text { () não vai ao banheiro ou equivalente } \\
\text { para eliminações fisiológicas }\end{array}$ & $\begin{array}{l}\text { Uso da privada: ir ao banheiro para eliminação } \\
\text { intestinal e de urina; limpar-se após a eliminação } \\
\text { e arrumar as roupas } \\
\text { () vai ao banheiro, se limpa e arruma as } \\
\text { roupas sem assistência (pode usar } \\
\text { objeto de apoio como bengala, andador } \\
\text { ou cadeira de rodas e pode usar penico } \\
\text { noturno ou comadre, esvaziando-o } \\
\text { de manhã) } \\
\text { () recebe assistência para ir ao banheiro, } \\
\text { ou se limpar, ou arrumar as roupas, após a } \\
\text { eliminação, ou para usar o penico noturno } \\
\text { ou comadre } \\
\text { () não vai ao quarto chamado "banheiro" } \\
\text { para o processo de eliminação }\end{array}$ \\
\hline
\end{tabular}


sofrendo influência de escolaridade ou gênero. Alguns examinadores sugeriram maior detalhamento do instrumento como, por exemplo: no item referente à continência, solicitou-se explicar o que é catéter e também acrescentar a variável "uso freqüente de fralda geriátrica". No item referente ao banho sugeriu-se acrescentar outras partes do corpo além daquelas já citadas. Os autores entenderam que tais modificações alterariam o instrumento original e mantiveram o texto.

Um total de 156 anciãos foram analisados por 4 aplicadores com a versão em português da Escala de Katz, sendo 57 com reavaliação no mesmo dia e 99 com 7 dias de intervalo (Tabela 3). No subgrupo em que o reteste foi feito no mesmo dia a concordância foi de 78,9\% (IC95\%: 66,1-88,6) e a concordância corrigida para o acaso (kappa) foi de 0,74 (IC95\%: 0,61-0,87). O kappa ponderado foi 0,91 (IC95\%: 0,86-0,96). A distribuição das diferenças no teste-reteste mostrou pequena assimetria: pontuação média na Escala de Katz igual a -0,11 (IC95\%: -0,25-0,04) sem significância estatística $(\mathrm{p}=0,1591)$.

Quando o reteste foi feito com sete dias de intervalo a concordância foi de 50,5\% (IC95\%: 40,3-60,7), o kappa foi 0,41 (IC95\%: 0,30-0,53) е o kappa ponderado 0,67 (IC95\%: 0,58-0,76). A distribuição das diferenças na pontuação no teste e reteste mostrou assimetria substancial média de 0,36 (IC95\%: 0,13-0,59) e estatisticamente significativa ( $\mathrm{p}=0,0021)$.

Quando a análise do reteste no mesmo dia desconsiderou o item "transferência" o kappa passou de 0,74 para 0,82. Com os dados do reteste com sete dias de intervalo, desconsiderando o item "banho", o kappa foi de 0,41 para 0,54 . Os demais itens não mostraram impacto substancial na confiabilidade da pontuação (Tabela 3 ).

Os itens no reteste no mesmo dia mostraram consistência interna com os dados dos observadores 1 e 2 (alfa $1=0,92$; alfa $2=0,91$ ) superior ao observado no reteste com sete dias de intervalo (alfa $1=0,80$; alfa $2=0,83$ ). A consistência aumentou ligeiramente quando a análise desconsiderou o item "alimentação".

A concordância entre os observadores em cada um dos itens da escala foi, de modo geral, maior quando teste e reteste foram feitos no mesmo dia (Tabela 4). A concordância corrigida foi substancialmente menor nos itens "continência", "vestir" e "banho".

Durante a etapa de análise da equivalência de medida surgiram situações que deram margem a dúvidas não solucionadas por consenso entre os pesquisadores, optando-se por contatar o autor do instrumento. As seguintes questões foram analisadas e interpretadas pelo Dr. Katz:
Tabela 3

Pontuação na Escala de Katz obtida por dois observadores independentes no mesmo dia e com sete dias de intervalo.

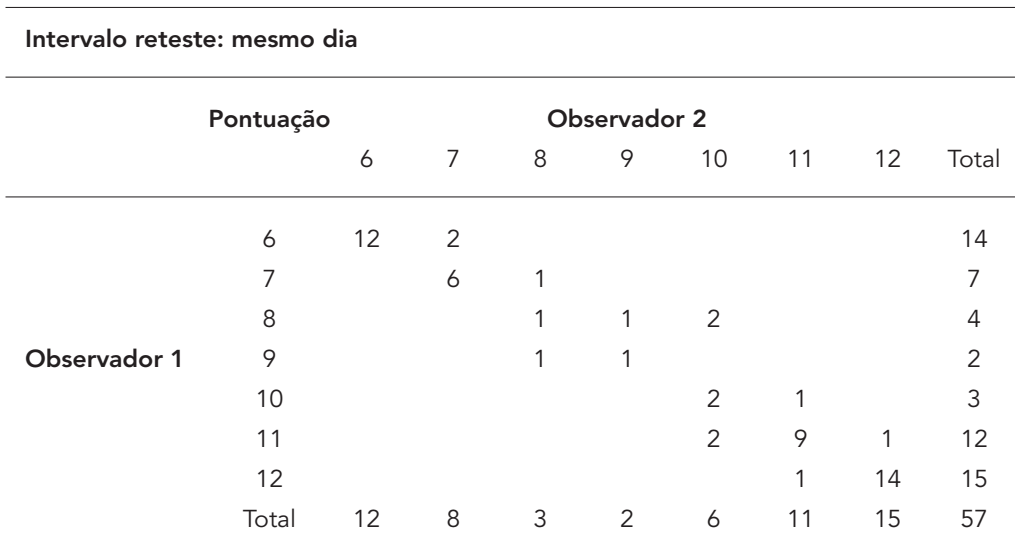

Intervalo reteste: 7 dias

\begin{tabular}{cccccccccc}
\hline & Pontuação & \multicolumn{7}{c}{ Observador 2 } \\
& & 6 & 7 & 8 & 9 & 10 & 11 & 12 & Total \\
\hline \multirow{5}{*}{ Observador 1 } & 6 & 7 & 2 & 2 & & & & & 11 \\
& 7 & 10 & 4 & & 1 & & & & 15 \\
& 8 & 1 & & 5 & 1 & 1 & 1 & & 9 \\
& 9 & & 5 & 1 & 2 & & & & 8 \\
& 10 & & 2 & 4 & 4 & 11 & 3 & 1 & 25 \\
& 11 & 1 & & & & 7 & 14 & & 22 \\
& 12 & & & & & & 2 & 7 & 9 \\
& Total & 19 & 13 & 12 & 8 & 19 & 20 & 8 & 99
\end{tabular}

Tabela 4

Concordância simples e corrigida para o acaso (índice kappa) entre observadores independentes, no mesmo dia ou com sete dias de intervalo, por item da Escala de Katz.

\begin{tabular}{lcccc}
\hline \multirow{2}{*}{ Item } & \multicolumn{2}{c}{ Concordância } & \multicolumn{2}{c}{ Kappa } \\
& Mesmo dia & 7 dias & Mesmo dia & $\mathbf{7}$ dias \\
\hline Alimentação & 0,98 & 0,96 & 0,96 & 0,80 \\
Continência & 0,96 & 0,84 & 0,92 & 0,66 \\
Transferência & 0,91 & 0,91 & 0,82 & 0,81 \\
Sanitário & 0,93 & 0,91 & 0,86 & 0,81 \\
Vestir & 0,91 & 0,82 & 0,81 & 0,50 \\
Banho & 0,98 & 0,70 & 0,96 & 0,40 \\
\hline
\end{tabular}

a) Um indivíduo que não consegue se vestir sozinho, em virtude de não alcançar uma prateleira alta para pegar as roupas - dependente;

b) Residente de instituição de longa permanência que utiliza cadeira de rodas sem freio e neces- 
sita de terceiros para segurar a mesma na hora da transferência-dependente;

c) Paciente que pode se banhar sozinho, mas, por insegurança, requer a presença de outra pessoa dentro do banheiro observando o banho - dependente;

d) Paciente demente que mantém a continência urinária e fecal, mas urina em locais impróprios, como por exemplo, a sala-de-estar - dependente;

e) Paciente colostomizado que é capaz de trocar e limpar a bolsa de colostomia sem ajuda - independente;

f) Paciente que deve ser conduzido para o banho em cadeira de rodas, mas é capaz de se banhar sozinho-dependente.

A caracterização do estado funcional é baseada no estado real e não na habilidade de realizar a tarefa sem auxílio de terceiros. Dessa forma, nas situações "a" e "b", os indivíduos serão considerados dependentes até que se resolvam os problemas ambientais.
Ser independente é ser capaz de desempenhar a função sem supervisão. Assim, um idoso que só toma banho quando há um parente presente dentro do banheiro, como no exemplo "c", é considerado dependente, apesar de ser capaz de realizar o ato sem auxílio direto. $\mathrm{O}$ mesmo se dá no modelo "d" em que o paciente é continente, mas precisa de controle externo para executar a ação nos locais adequados. Por outro lado, na condição "e", o indivíduo incontinente que consegue manusear a bolsa de colostomia ou a fralda é considerado independente.

A última dúvida gerada durante a pesquisa, descrita em "f", surgiu pela dificuldade de discriminar o tipo de dependência. Houve acordo em relação à dependência na transferência e discordância em relação ao banho. $\mathrm{O}$ autor do instrumento original considerou o paciente dependente, pois ele necessita de auxílio para o desempenho da função.

O cumprimento de todas as etapas exigidas para a adaptação transcultural originou a versão em português da EIAVD (Tabelas 5 e 6).

Escala de Independência em Atividades da Vida Diária: a escala de independência em atividades da vida diária é baseada numa avaliação da independência ou dependência funcional de pacientes ao tomar banho, vestir-se, ir ao vaso sanitário, transferir-se, manter-se continente e alimentar-se.

\section{Tomar banho}

(leito, chuveiro ou banheira)

ou toma banho sozinho

Vestir-se

Uso do vaso sanitário

Continência

Alimentação
Requer ajuda somente para lavar uma única parte do corpo (como as costas ou membro deficiente) ou não toma banho sozinho

Pega as roupas nos armários e gavetas, veste-as, coloca órteses ou próteses, manuseia fechos.

Exclui-se o ato de amarrar sapatos

Vai ao vaso sanitário, senta-se e levanta-se do vaso ajeita as roupas, faz a higiene íntima (pode usar comadre ou similar somente à noite e pode ou não estar usando suportes mecânicos)

Deita-se e sai da cama sozinho, senta e se levanta da cadeira sozinho (pode estar usando objeto de apoio)

Micção e evacuação inteiramente autocontroladas
Requer ajuda para lavar mais de uma parte do corpo, ou para entrar ou sair da banheira,

Veste-se apenas parcialmente ou não se veste sozinho

Usa comadre ou similar, controlado por terceiros, ou recebe ajuda para ir até o vaso sanitário e usá-lo

Requer ajuda para deitar na cama ou sentar na cadeira, ou para levantar-se; não faz uma ou mais transferências Incontinência parcial ou total para micção ou evacuação; controle parcial ou total por enemas e/ou catéteres; uso de urinóis ou comadre controlado por terceiros

Requer ajuda para levar a comida do prato (ou de seu equivalente) à boca; não come nada ou recebe alimentação parenteral

Nota: independência significa a realização dos atos citados sem supervisão, orientação ou assistência pessoal ativa, exceto nos casos especificamente descritos abaixo. Esta avaliação é baseada no real desempenho e não na habilidade. Um paciente que se recusa a executar uma função é considerado como não a tendo executado, mesmo que se julgue ser ele capaz. 
Ficha de avaliação: para cada área de funcionamento listada abaixo assinale a descrição que se aplica (a palavra "ajuda" significa supervisão, orientação ou auxílio pessoal).

Tomar banho (leito, banheira ou chuveiro)

( ) não recebe ajuda (entra e sai da banheira sozinho, se este for o modo habitual de tomar banho)

( ) recebe ajuda para lavar apenas uma parte do corpo (como, por exemplo, as costas ou uma perna)

( ) recebe ajuda para lavar mais de uma parte do corpo, ou não toma banho sozinho

Vestir-se (pega roupas, inclusive peças íntimas, nos armários e gavetas, e manuseia fechos, inclusive os de órteses e próteses, quando forem utilizadas)

( ) pega as roupas e veste-se completamente, sem ajuda

( ) pega as roupas e veste-se sem ajuda, exceto para amarrar os sapatos

( ) recebe ajuda para pegar as roupas ou vestir-se, ou permanece parcial ou completamente sem roupa

Uso do vaso sanitário (ida ao banheiro ou local equivalente para evacuar e urinar; higiene íntima e arrumação das roupas)

( ) vai ao banheiro ou local equivalente, limpa-se e ajeita as roupas sem ajuda (pode usar objetos para apoio como

bengala, andador ou cadeira de rodas e pode usar comadre ou urinol à noite, esvaziando-o de manhã)

( ) recebe ajuda para ir ao banheiro ou local equivalente, ou para limpar-se, ou para ajeitar as roupas após evacuação

ou micção, ou para usar a comadre ou urinol à noite

( ) não vai ao banheiro ou equivalente para eliminações fisiológicas

Transferência

( ) deita-se e sai da cama, senta-se e levanta-se da cadeira sem ajuda (pode estar usando objeto para apoio, como

bengala ou andador)

( ) deita-se e sai da cama e/ou senta-se e levanta-se da cadeira com ajuda

( ) não sai da cama

Continência

( ) controla inteiramente a micção e a evacuação

( ) tem "acidentes" ocasionais

( ) necessita de ajuda para manter o controle da micção e evacuação; usa cateter ou é incontinente

Alimentação

( ) alimenta-se sem ajuda

( ) alimenta-se sozinho, mas recebe ajuda para cortar carne ou passar manteiga no pão

( ) recebe ajuda para alimentar-se, ou é alimentado parcialmente ou completamente pelo uso de catéteres ou fluidos intravenosos

Interpretação (Katz \& Apkom 26):

0 : independente em todas as seis funções; 1 : independente em cinco funções e dependente em uma função; 2: independente em quatro funções e dependente em duas; 3 : independente em três funções e dependente em três; 4: independente em duas funções e dependente em quatro; 5 : independente em uma função e dependente em cinco funções; 6: dependente em todas as seis funções.

\section{Discussão}

Utilizando-se a estratégia de Herdman et al. 25 , observamos discrepâncias entre o instrumento original e a retradução, havendo, assim, oportunidades de melhora na tradução, que foram discutidas abertamente pelos pesquisadores. Embora longo, o procedimento sistemático foi necessário para garantir a produção de uma versão com equivalência lingüística.

O fato de não haver termos completamente alterados na análise do significado referencial deve-se, em nossa opinião, à simplicidade do ins- trumento, que avalia atividades essenciais para o autocuidado, naturalmente adquiridas à medida que o ser humano se desenvolve. Em razão disso pode-se prever que a interpretação das mesmas sofra poucas influências entre as culturas. Todos os itens analisados são pertinentes às duas culturas e os termos apresentam similaridade em ambos os idiomas, caracterizando uma tradução simétrica 16. Não obstante, a expressão "sponge bath", traduzida como "banho de esponja” por um dos tradutores, e "banho de leito" por outro, mostrou a importância de haver um ou mais médicos envolvidos no processo, influenciando 
positivamente o resultado final, já que a primeira expressão não tem significância clínica em nosso meio.

Em relação à equivalência operacional, embora o instrumento original tenha sido elaborado por meio da observação direta de pacientes internados, a escala vem sendo largamente usada na forma de entrevista ambulatorial, apresentando resultados semelhantes à análise do desempenho individual 30,31,32,33. Igualmente a esses estudos, encontramos equivalência operacional aplicando o instrumento tanto em nível ambulatorial quanto hospitalar, por profissionais não bilíngües que lidam com idosos. Com respeito à compreensão do questionário, nenhum dos entrevistados apresentou dificuldades para respondê-lo. Em nossa opinião, isso se deve à formulação simples e objetiva do instrumento original, observação já feita por outros autores 34 .

A concordância interobservador corrigida para o acaso alcançada com esta versão do instrumento foi substancial no reteste no mesmo dia e moderada no reteste com sete dias de intervalo. Considerando a concordância parcial através do kappa ponderado, a concordância foi quase perfeita no reteste do mesmo dia e substancial no reteste com sete dias de intervalo. Neste subgrupo, a discordância entre os observadores não só foi maior como também indicou tendência do observador 1 de atribuir pontuações maiores do que o observador 2. É possível que a convergência dos observadores possa ser aumentada por meio de instruções com vistas a ampliar a homogeneidade na aplicação dos itens, particularmente aqueles que mostraram maior influência na concordância, como "banho", "vestir" e "transferência”. Para ambos os observadores, o instrumento indicou maior coerência interna no subgrupo retestado no mesmo dia. Os níveis do coeficiente alfa de Cronbach alcançados podem ser tomados como evidência empírica da valida- de do constructo independência nas atividades da vida diária conforme avaliado pela escala. Sua validade de conteúdo pode ser defendida considerando-se a convergência entre geriatras sobre os elementos componentes do que tem sido chamado de independência nas atividades da vida diária. A validade de critério não foi avaliada diretamente, mas pode ter sido afetada pelas limitações observadas na reprodutibilidade.

Neste trabalho houve grande variabilidade no item "banho" nas avaliações entre os observadores. Esse fato poderia ser explicado pelo sintetismo da escala, levando o respondedor a interpretar os fatos de forma diferente. Um instrumento mais completo, com maior detalhamento, permitiria a escolha de graus intermediários de dependência.

A falta de coincidência nas respostas em outros itens pode ter ocorrido devido ao intervalo de tempo entre as entrevistas, levando o respondedor a fornecer diferentes informações, como já observado por Applegate et al. 35. Descarta-se a possibilidade de ter havido modificações na capacidade em realizar o ato, pois no espaço de sete dias, na ausência de intercorrências clínicas, torna-se pouco provável o surgimento de mudanças significativas na capacidade funcional.

Concluímos que o método de Herdman et al. 25 permitiu obter uma versão em português do EIAVD fiel ao original em inglês, pois a retradução se aproximou do original, e onde havia diferenças, estas foram corrigidas. A aplicação em campo da versão em português confirmou a funcionalidade da escala original. A despeito disso, há situações que podem gerar dúvidas, necessitando de desdobramentos durante a aplicação do questionário. Sugerimos um treinamento com exercícios para a completa compreensão do que seja supervisão, orientação ou assistência pessoal ativa, a fim de se identificar corretamente o estado funcional. 


\section{Resumo}

Desenvolvimento e análise do desempenho de uma adaptação transcultural para o português da Escala de Katz de independência em atividades da vida diária. Duas traduções e duas retrotraduções analisaram as equivalências conceitual, de itens e semântica para a escolha da versão final. A equivalência operacional foi avaliada em um estudo piloto, testando-se a confiabilidade e a consistência interna da versão adaptada por meio de reteste no mesmo dia em 156 pacientes ou após sete dias da primeira entrevista. A resolução de diferenças sutis em alguns itens levou à equivalência cultural. A versão final foi considerada fácil de se entender e de aplicar. A concordância corrigida para o acaso (kappa ponderado) foi de 0,91. O alfa de Chronbach variou de 0,80 a 0,92. A versão em português da Escala de Katz de independência em atividades da vida diária, completamente desenvolvida e testada, provou ser equivalente à original em inglês. Os itens apresentaram consistência interna e as taxa foram confiáveis.

Atividades Cotidianas; Reprodutibilidade dos Testes; Idoso

\section{Colaboradores}

V. T. S. Lino e S. R. M. Pereira participaram do grupo T1, pesquisa de campo para análise de confiabilidade, seleção dos termos para elaboração final da versão em português e escreveram o artigo. L. A. B. Camacho realizou o cálculo do tamanho da amostra, a análise da equivalência semântica entre o original e cada uma das retraduções (R1 e R2) e análise da confiabilidade. S. T. Ribeiro Filho e S. Buksman participaram do grupo $\mathrm{T} 1$, pesquisa de campo para análise de confiabilidade e seleção dos termos para elaboração final da versão em português.

\section{Referências}

1. Costa EFA, Porto CC, Almeida JC, Cipullo JP, Martin JFV. Semiologia do idoso. In: Porto CC, organizador. Semiologia médica. Rio de Janeiro: Editora Guanabara Koogan; 2002. p. 166-97.

2. Chaimowicz F. Envelhecimento populacional brasileiro. In: Chaimowicz F, organizador. Os idosos brasileiros no século XXI: demografia, saúde e sociedade. Belo Horizonte: Postgraduate; 1998. p. 3342.

3. Grundy EMD. Epidemiology of aging. In: Tallis RC, Fillit HM, editors. Brocklehurst's textbook of geriatric medicine and gerontology. Edinburgh: Churchill Livingstone; 2003. p. 3-20.

4. Evans BC, Crogan NL. Building a scientific base for nutrition care of Hispanic nursing home residents. Geriatr Nurs 2006; 27:273-9.

5. Einarsson U, Gottberg K, Fredrikson S, von Koch L, Holmqvist LW. Activities of daily living and social activities in people with multiple sclerosis in Stockholm County. Clin Rehabil 2006; 20:543-51.

6. Esbensen BA, Osterlind K, Hallberg IR. Quality of life of elderly persons with cancer: a 3-month follow-up. Cancer Nurs 2006; 29:214-24.

7. Avila-Funes JA, Garant MP, Aguilar-Navarro S. Relationship between determining factors for depressive symptoms and for dietary habits in older adults in Mexico. Rev Panam Salud Pública 2006; 19:321-30.
8. von Heideken Wågert P, Gustavsson JM, LundinOlsson L, Kallin K, Nygren B, Lundman B, et al. Health status in the oldest old: age and sex differences in the Umeå 85+ Study. Aging Clin Exp Res 2006; 18:116-26.

9. Denny SD, Kuchibhatla MN, Cohen HJ. Impact of anemia on mortality, cognition, and function in community-dwelling elderly. Am J Med 2006; 119:327-34.

10. Hill J, Fillit H, Thomas SK, Chang S. Functional impairment, healthcare costs and the prevalence of institutionalisation in patients with Alzheimer's disease and other dementias. Pharmacoeconomics 2006; 24:265-80.

11. Multidisciplinary studies of illness in aged persons. II. A new classification of functional status in activities of daily living. J Chronic Dis 1959; 9:5562.

12. Katz S, Ford A, Moskowitz RW, Jackson BA, Jaffe MW. Studies of illness in the aged. The index of ADL: a standardized measure of biological and psychosocial function. JAMA 1963; 12:914-9.

13. Dowd S, Davidhizar R. Opening up to the Katz Index. Elder Care 1999; 11:9-12.

14. Ramos LR, Rosa TEC, Oliveira ZMA. Perfil do idoso em área metropolitana na região sudeste do Brasil: resultados de inquérito domiciliar. Rev Saúde Pública 1993; 27:87-94. 
15. Asberg KH, Sonn U. The cumulative structure of personal and instrumental ADL: a study of elderly people in a health service district. Scand J Rehabil Med 1989; 21:171-7.

16. Brorsson B, Asberg KH. Katz Index of independence in ADL: reliability and validity in short-term care. Scand J Rehabil Med 1984; 16:125-32.

17. Veras RP. País jovem com cabelos brancos: a saúde do idoso no Brasil. Rio de Janeiro: Editora RelumeDumará; 1994.

18. Fundação João Pinheiro. População idosa em Minas Gerais e políticas de atendimento. Belo Horizonte: Fundação João Pinheiro; 1993.

19. Rassi EPM. Indicadores de saúde em idosos: o papel da unidade básica de saúde. Gerontologia 1994; 2:75-81.

20. Moraes CL, Reichenheim ME. Cross-cultural measurement equivalence of the Revised Conflict Tactics Scales (CTS2) Portuguese version used to identify violence within couples. Cad Saúde Pública 2002; 18:783-96.

21. Hilton A, Strutkowiski M. Translating instruments into other languages: development and testing processes. Cancer Nurs 2002; 25:1-7.

22. Ferraz MB, Oliveira LM, Araújo PMP, Atra E, Tugwell P. Crosscultural reliability of the physical ability dimension of the health assessment questionnaire. J Rheumatol 1990; 17:813-7.

23. Guillemin F, Bombardier C, Beaton D. Cross-cultural adaptation of health-related quality of life measures: literature review and proposed guidelines. J Clin Epidemiol 1993; 46:1417-32.

24. Reichenheim ME, Moraes CL, Hasselmann MH. Equivalência semântica da versão em português do instrumento Abuse Assessment Screen para rastrear a violência contra a mulher grávida. Rev Saúde Pública 2000; 34:610-6.
25. Herdman M, Fox-Rushby J, Badia X. A model of equivalence in the cultural adaptation oh HRQL instruments: the universalist approach. Qual Life Res 1998; 7:323-35.

26. Katz S, Akpom CA. A measure of primary sociobiological functions. Int J Health Serv 1976; 6:493-508.

27. Cohen J. A coefficient of agreement for nominal scales. Educ Psychol Meas 1960; 20:37-46.

28. Cohen J. Weighted kappa: nominal scale agreement with provisions for scales disagreement of partial credit. Psychol Bull 1968; 70:213-20.

29. Landis JR, Koch GG. The measurement of observer agreement for categorical data. Biometrics 1977; 33:159-74.

30. Ferrell BA, Stein WM, Beck JC. The Geriatric Pain Measure: validity, reliability and factor analysis. J Am Geriatr Soc 2000; 48:1669-73.

31. Rubin CD, Sizemore MT, Loftis PA, de Mola NL. A randomized, controlled trial of outpatient geriatric evaluation and management in a large public hospital. J Am Geriatr Soc 1993; 41:1023-8.

32. Reuben DB, Siu AL, Kimpau S. The predictive validity of self-report and performance-based measures of function and health. J Gerontol 1992; 47: M106-10.

33. Ware Jr. JE, Keller SD, Gandek B, Brazier, JE, Sullivan M. Evaluating translations of health status questionnaires. Int J Technol Assess Health Care 1995; 11:525-51.

34. Elola-Olasco CM, Rei E, Artalejo FR, Locke III GR, Diaz-Rubio M. Adaptation and validation of gastroesophageal reflux questionnaire for use on a Spanish population. Rev Esp Enferm Apar Dig 2002; 94:752-8.

35. Applegate WB, Blass JP, Williams TF. Instruments for the functional assessment of older patients. $\mathrm{N}$ Engl J Med 1990; 322:1207-14.

Recebido em 31/Ago/2006

Versão final reapresentada em 31/Mai/2007

Aprovado em 25/Jun/2007 